\title{
ON THE USE OF CORRESPONDENCE ANALYSIS TO LEARN SEED ONTOLOGIES FROM TEXT
}

\author{
Davide Eynard, Fabio Marfia and Matteo Matteucci \\ Dept. of Electronics and Information, Politecnico di Milano, Via Ponzio 34/5, I-20133, Milan, Italy \\ \{davide.eynard, matteo.matteucci\}@polimi.it,fabio.marfia@stud.polimi.it
}

Keywords: Ontology learning from text, Knowledge extraction, Correspondence analysis, Machine learning.

\begin{abstract}
In the present work we show our approach to generate hierarchies of concepts in the form of ontologies starting from free text. This approach relies on the statistical model of Correspondence Analysis to analyze term occurrences in text, identify the main concepts it refers to, and retrieve semantic relationships between them. We present a tool which is able to apply different methods for the generation of ontologies from text, namely hierarchy generation from hierarchical clustering representation, search for Hearst Patterns on the Web, and bootstrapping. Our evaluation shows that the precision in the generation of hierarchies of the tool is attested to be around $60 \%$ for the best automatic approach and around $90 \%$ for the best human-assisted approach.
\end{abstract}

\section{INTRODUCTION}

In the last years there has been a considerable increase in research on knowledge-based systems, especially in the context of the Semantic Web. However these systems, as long as they number between their objectives something more than supplying trivial functionalities, suffer in their development process of the so-called knowledge acquisition bottleneck: creating large, usable, expandable and valid representations of semantics about a specific domain of interest represents the most time-consuming task of the whole project. The cause of this stands in the fact that, being these structures supposed to represent a collection of semantics previously unknown to machines, they need to be manually annotated by domain experts.

Actually, there is already plenty of information available on the Internet which could be used to teach a machine about virtually any domain of knowledge: this information is stored in the form of collections of Web pages, large corpora of documents, databases, and so on. These repositories, however, cannot be directly consumed by a machine as they contain unstructured information according to any standard model for knowledge representation.

One of the main issues in ontology building is that two different kinds of expertise are required: the knowledge of the domain that has to be described, and the ability to encode the ontology in a machineinterpretable format. Easing this task means either making semantic technologies more accessible to domain experts or providing structured information about a domain to ontology experts. Focusing mainly on the second part of this problem, our work provides an alternative to the manual generation of ontologies from scratch: automatically extract candidate concepts and relations from text and suggest a seed ontology as a first, approximate representation of the domain knowledge. This ontology can then be modified (possibly correcting wrong information) and expanded, reducing considerably the time required for the formalization of the domain knowledge.

The main challenge we face in our tool is the so called Ontology Learning from Text: free text is a repository of unstructured knowledge, and this requires researchers to adopt original heuristics in order to extract structured semantics. These heuristics often return inaccurate results, and have to be modified and validated by experts of the domain. Our work aims at increasing the accuracy of these methods, relying on different techniques during the main stages of ontology learning from text:

- the extraction of the most relevant concepts is performed according to their Information Gain with respect to a reference corpus of documents;

- subsumption relationships between the identified concepts are built using three different algorithms (hierarchy generation from hierarchical clustering representation, search for Hearst Patterns on the 
Web, and bootstrapping);

- the Correspondence Analysis framework has been employed for the computation of distributional similarity between terms, which is then used as a basis to extract different types of relationships.

In Section 2 we present the main approaches to the problem of the extraction of concept hierarchies that have been investigated in the context of our project. In Section 3 we explain in detail the steps of our solution for the extraction of concept hierarchies from free text. In Section 4 we present a summary of the results for the tests we performed on the different plugins of our tool. In Section 5 we draw our conclusions and summarize directions of our future work.

\section{ONTOLOGIES FROM TEXT}

The extraction of ontologies from text can be supervised by a human expert or use any sort of structured data as an additional source. In the former case, we are speaking of assisted or semi-automatic learning; in the latter, we refer to oracle guided learning; if the algorithm makes no use of structured sources or human help, it is considered an automatic learner. As an orthogonal definition, when the objective of the algorithm is the expansion of a pre-constructed ontology we talk about bootstrapping instead of learning.

A large number of methods for the ontology learning from text are based on the same conceptual approach defined Distributional Similarity. It is based on Harris' Distributional Hypothesis (Harris, 1968):

"Words are similar to the extent that they share similar context”.

The idea is to analyze the co-occurrence of words in the same sentence, paragraph, document, or other types of context to infer context similarity. The more two words are similar in their distribution over the contexts, the more they are expected to be semantically similar, and the more a potential directed relation is expected to stand between them.

In Distributional Similarity approaches, concepts are extracted and organized using some representation according to their distributional similarity. Then, different methods can be used to identify relationships between neighbors. ASIUM (Faure D., 1998), for example, is a software for the generation of concept hierarchies that uses as context the verb of the sentence where a concept appears and the syntactical function (i.e., subject, object, or other complements) of the concept itself. This tool presents semantically similar words to the user that can then suggest trough an interface their hierarchical organization, thus leaving relations discovery to the user.

Caraballo (Caraballo, 1999) presents an approach to build a hierarchy of concepts extracted from a corpus of articles from the Wall Street Journal, with the parser described in (Caraballo and Charniak, 1998). That model uses as context the paragraph in which the terms appear, while for the generation of the hierarchy it looks in the text for the so-called Hearst Patterns (Hearst, 1992). An example for such a pattern is " $t_{1} \mathrm{~s}$, such as $t_{2} \ldots$ ", whose occurrence suggests that term $t_{2}$ is a hyponym ${ }^{1}$ of term $t_{1}$. A further different approach is the Learning by Googling one: Hearst patterns can not only be found within document corpora, but they can also be searched on the Web. PANKOW (Cimiano P., 2004), for instance, is a software that looks for these patterns on Google and decides, according to the number of results returned by the engine, whether a subsumption relation can be confirmed or denied.

Another model is presented by Fionn Murtagh in (Murtagh, 2005) and (Murtagh, 2007). This is a Distributional Similarity approach that relies on Correspondende Analysis (a multivariate statistical technique developed by J.-P. Benzcri in the '60s (Benzcri, 1976)) to calculate the semantic similarity between concepts. The generation of the hierarchy starts from the assumption that terms appearing in more documents are more general than others, and the solution of Murtagh places them in a higher position in the hierarchy.

A strongly different approach from Distributional Similarity is Formal Concept Analysis, as described by Philipp Cimiano (Cimiano, 2006). It is based on different assumptions and largely relies on Natural Language Processing (NLP) algorithms. The idea in FCA is to identify the actions that a concept can do or undergo. Words are organized in groups according to the actions they share; then groups are ordered in a hierarchy always according to these actions (i.e. the group of entities that can run and eat and group of entities that can fly and eat are put together under the more general group of entities that can eat). Finally the user is asked to label every node of the formed hierarchy (or other automatic methods can be used to perform this operation), and the final hierarchy is obtained. The paper also describes an algorithm which generates hierarchies from text by using, as a sort of prompter, a pre-constructed ontology. What the model obtains is not an extension of the pre-existent ontology (as for bootstrapping methods), but a new

\footnotetext{
${ }^{1}$ When a $i s-a$ relationship in an ontology starts from term $t_{1}$ and arrives at term $t_{2}, t_{1}$ is defined as a hyponym of $t_{2}$, while $t_{2}$ is defined as a hypernym of $t_{1}$.
} 
and independent one, not necessarily containing all the entities and relationships of the starting model.

For what concerns bootstrapping approaches, many methods have been developed which are based on distributional similarity ((Hearst M., 1993), (Schutze, 1993), (Alfonseca E., 2002) and (Maedche A., 2003)). Their common approach is to evaluate from a corpus of documents the similarity between a word to be added in the ontology and other words already present in it. Then the algorithms put the new concept as a son of the concept in the ontology that has the more similar children, according to the similarity information that has been computed.

\section{THE EXTRACTION TOOL}

For our work, we decided to be based on the conceptual model presented by Fionn Murtagh in (Murtagh, 2005) and (Murtagh, 2007). The main steps of our approach are depicted in Figure 1.

\subsection{Data Indexing}

To reduce the complexity of the problem, we start by selecting a set of terms that can be considered as the more relevant for the document corpus. To do this we start from two corpora of documents: the training corpus, which is used as a referential corpus by the procedure of identification of the relevant terms, and the test corpus, that is the main corpus from which the terms are to be extracted and the ontology has to be learned.

To allow our application to rapidly handle the documents and the terms contained within them, our first task is to index the two document corpora. To perform this operation we used Lucene ${ }^{2}$, which takes care of tokenization, indexing, and stopwords filtering. The indexing process is usually very time-consuming, so users are allowed to save the indexes generated and reuse them across different executions.

\subsection{Data Filtering}

Moreover, during the indexing phase, filters can be applied in order to select terms according to specific needs: two different filters (one based on Wordnet and the other on NLP algorithms) can be used to discard everything but the nouns, while another one automatically discards terms composed by less than $n$ letters (usually, very short terms rarely represent relevant concepts, even if there are some exceptions such as with acronyms).

\footnotetext{
${ }^{2} \mathrm{http}: / /$ lucene.apache.org
}

\subsection{Identification of Relevant Terms in the Test Corpus}

For every term appearing in the test corpus the score of Information Gain is computed. Information Gain measure is based on the concept of entropy. In our case, we want to calculate how the total entropy changes for the two training and test corpora by knowing that a specific term is more or less common within these sets. We can identify the $P(i)$ function as the probability for a document to appear in a corpus $i$, computed as the number of the documents in $i$ divided by the total number of documents:

$$
p(i)=\frac{\text { document } \operatorname{In}(i)}{\text { totalDocuments }}
$$

Where $i$ can be training or test. Our entropy measure for a group of documents $D_{g}$ distributed in the two corpora is measured as:

$$
H_{D_{g}}=-\sum_{i} p(i) \cdot \log (p(i))
$$

We identify three different groups of documents:

- the group of all documents, $D_{\text {total }}$;

- documents presenting the term of interest $t$ in them, $D_{t}$;

- documents not presenting the term of interest $t$ in them, $D_{\neg t}$.

Information Gain score of term $t$ is then calculated as:

$$
I G(t)=H_{D_{\text {total }}}-\frac{\left|D_{t}\right|}{\left|D_{\text {total }}\right|} H_{D_{t}}-\frac{\left|D_{\neg t}\right|}{\left|D_{\text {total }}\right|} H_{D_{\neg t}}
$$

Information Gain returns low values for terms that are very common in both training and test corpora. Terms that, instead, are very common in just one of the two corpora make Information Gain return high results. In this context, the training corpus has the role of a reference for the test corpus: terms that are frequent in test corpus can both be characterizing terms of the corpus, or very useless terms as conjunction, common adverbs, common verbs. If a term $t$ is very frequent also in the training corpus, which refers to a topic different from the test corpus, $t$ is supposed to be a useless term, and, in fact, it will receive a lower IG value. Vice versa, if $t$ is very frequent in test corpus, but not in training corpus it is supposed to be a characterizing term of the test corpus, and in fact, it will receive a higher IG value.

At the end, a set of $n$ (with $n$ specified by the user) relevant terms is collected, as the top $n$ terms of the IG rank. 

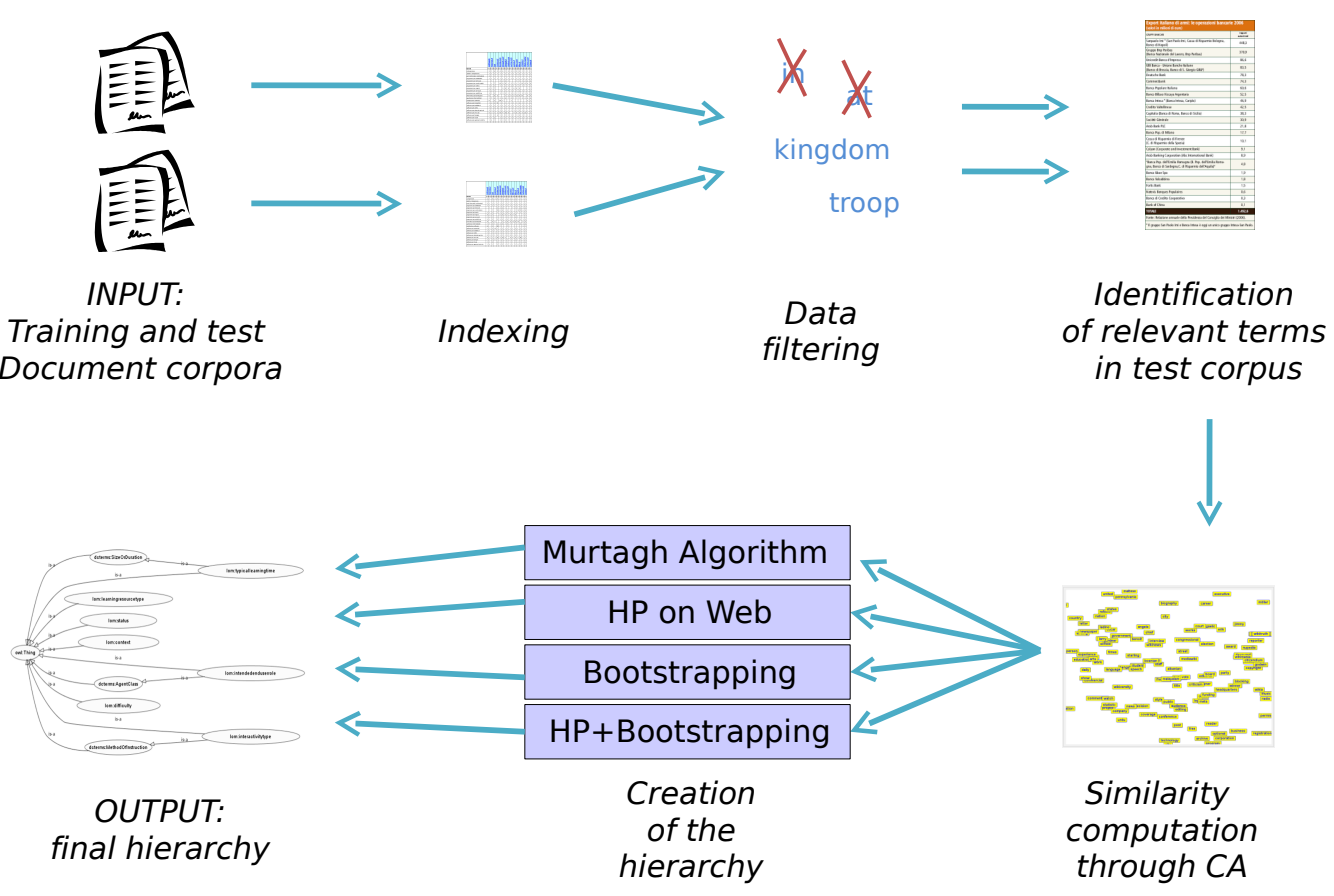

Figure 1: Main steps of our approach for ontology learning from text.

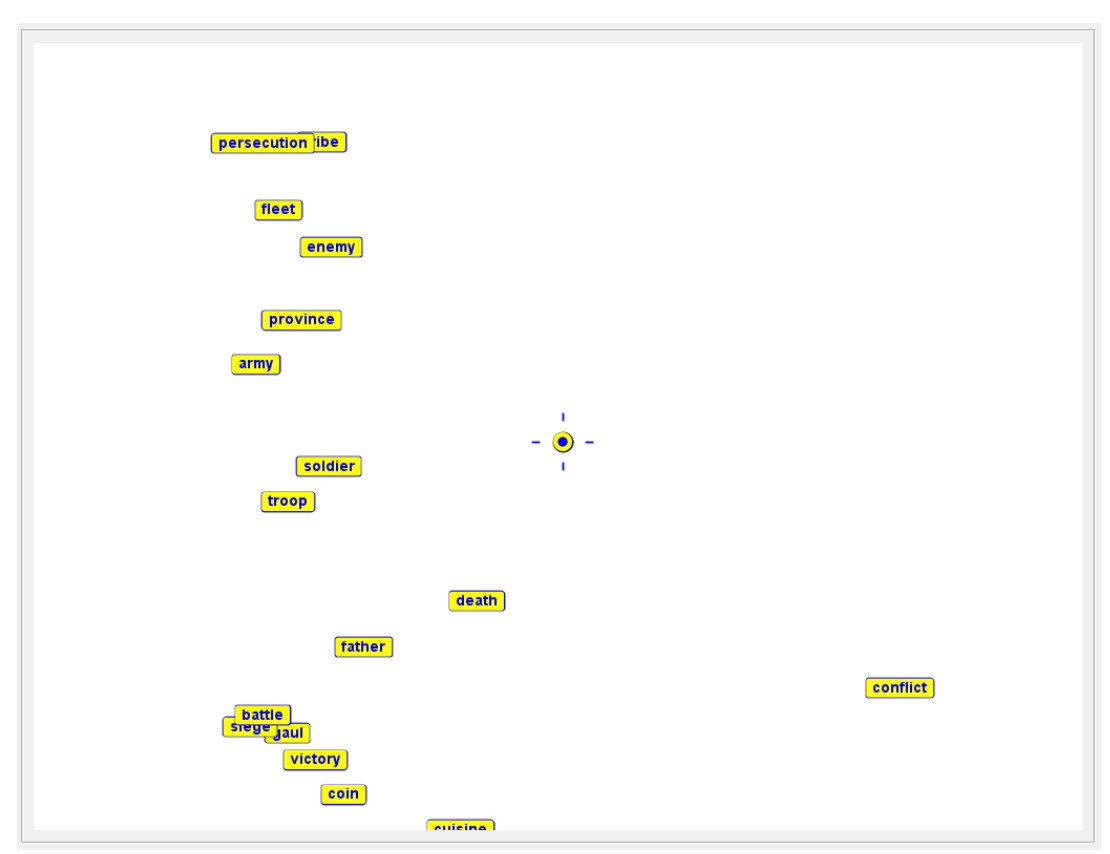

Figure 2: An example of Correspondence Analysis 2-dimensional projection, from a corpus of documents about the Roman Empire.

\subsection{Similarity Computation through Correspondence Analysis}

The approach of Correspondence Analysis (Murtagh, 2005) is used to project the relevant terms in a k-dimensional Euclidean space $^{3}$ according to

\footnotetext{
${ }^{3} k$ can be chosen at will by the user: the more the dimensions, the higher the precision of the distances in the representation.
} 
their distributional behavior over the documents. Correspondence Analysis starts from a (test documents $) \times($ relevant terms $)$ matrix, where the cell $n_{i, j}$ holds the number of occurrences of the $j$ th term in the $i$ th document, as, for example:

\begin{tabular}{|l|ccc|}
\hline & caligula & city & group \\
\hline D1 & 60 & 12 & 60 \\
D2 & 20 & 54 & 5 \\
D3 & 32 & 3 & 2 \\
D4 & 1 & 2 & 5 \\
\hline
\end{tabular}

In order to create the Euclidean space, Correspondence Analysis operates over the matrix different transformations. The first step is to calculate the grand total of the individual observations and divide each number in the cells for this grand total. This is done in order to have a matrix $M_{\text {prob }}$ expressing in the $n_{i, j}$ cell the probability of co-occurrence of the two $(i, j)$ modalities (i.e., documents and terms). In this simple example, the grand total is $\mathbf{3 7 6}$, and the probability matrix results:

\begin{tabular}{|c|ccc|}
\hline$M_{\text {prob }}$ & caligula & city & group \\
\hline D1 & 0.159 & 0.031 & 0.159 \\
D2 & 0.053 & 0.143 & 0.013 \\
D3 & 0.085 & 0.007 & 0.005 \\
D4 & 0.002 & 0.005 & 0.332 \\
\hline
\end{tabular}

Then the sums of the values of each row and each column are computed; we call the sum of the values of the $i$ th row $F_{i}$ and the sum of the values of the $j$ th column $F_{j}$. These are the marginal distributions of the two modalities:

\begin{tabular}{|c|ccc|c|}
\hline$M_{\text {prob }}$ & eat & city & group & $F_{i}$ \\
\hline D1 & 0.159 & 0.031 & 0.159 & $35 \%$ \\
D2 & 0.053 & 0.143 & 0.013 & $21 \%$ \\
D3 & 0.085 & 0.007 & 0.005 & $10 \%$ \\
D4 & 0.002 & 0.005 & 0.332 & $34 \%$ \\
\hline$F_{j}$ & $30 \%$ & $19 \%$ & $51 \%$ & \\
\hline
\end{tabular}

Being $\sum_{n=0}^{i} F_{i}=1$ and $\sum_{n=0}^{j} F_{j}=1$, we prefer to report $F_{i}$ and $F_{j}$ as percentages, every percentage represents the contribution of the $i$ th or $j$ th modality to the total of the occurrences. Now we proceed by generating the so-called matrix of column profiles. Let us consider the columns of our $M_{\text {prob }}$ matrix; the algorithm divides every $n_{i, j}$ probability by the $F_{j}$ value. What we obtain is the matrix whose columns are called column profiles:

\begin{tabular}{|c|c|c|c|c|}
\hline$M_{\text {colprof }}$ & caligula & city & group & $F_{i}$ \\
\hline D1 & $53 \%$ & $17 \%$ & $31 \%$ & $35 \%$ \\
D2 & $18 \%$ & $76 \%$ & $3 \%$ & $21 \%$ \\
D3 & $28 \%$ & $4 \%$ & $1 \%$ & $10 \%$ \\
D4 & $1 \%$ & $3 \%$ & $65 \%$ & $34 \%$ \\
\hline
\end{tabular}

Column profiles are a very important element of Correspondence Analysis because they represent the pure distributional behavior of column modalities (the terms, in our case), independently from the original amount of occurrences we were dealing with.

The last $F_{i}$ column represents the average behavior of the different column profiles. The divergences of the single column profiles from this average profile can be measured with the $\chi^{2}$ test of independence, and the $\chi^{2}$ distance between the $l$ column profile and $k$ column profile can be computed as:

$$
\chi^{2}(l, k)=\sqrt{\sum_{j} \frac{\left(n_{l, j}-n_{k, j}\right)^{2}}{F_{j}}} .
$$

The sum of all the $\chi^{2}$ tests applied to all column profiles in respect to the average profile represents the total inertia of the matrix in respect to his columns. Inertia represents the total amount of divergence of the column profiles from the assumption of independence. The higher is this number, the higher is the probability of an interdependence between rows and columns. There is also something more to say about the $\chi^{2}$ distance between two different column profiles: the obtained value represents how much two different rows diverge in their distributional behavior; the more similar this behavior is, the more there should be a similarity between the entities represented by the two columns (in our case these entities are the terms appearing in documents).

What Correspondence Analysis does, starting from the column profiles matrix, is to provide a summary representation of the similarities between the column modalities. In order to do so we project them into an Euclidean space of $k$ dimensions, where:

$$
k=\min (i-1, j-1)
$$

This space has the following properties:

- the Euclidean distance between the column modalities in this space of representation is exactly equal to the $\chi^{2}$ distance between their column profiles; calling the $\alpha$ th dimension of the $j$ th column $F_{\alpha}(j)$ we can state:

$$
\begin{aligned}
\chi^{2}(l, k) & =\sqrt{\sum_{i} \frac{\left(n_{i, l}-n_{i, k}\right)^{2}}{F_{i}}} \\
& =\sqrt{\sum_{\alpha}\left(F_{\alpha}(l)-F_{\alpha}(k)\right)^{2}}
\end{aligned}
$$

- the origin of the axis is placed in the barycenter of the different column profiles in respect to the $\chi^{2}$ distance measure, that is, as we said, the average column profile 


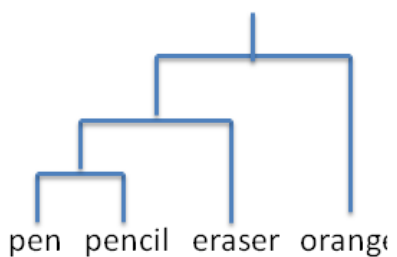

$A$

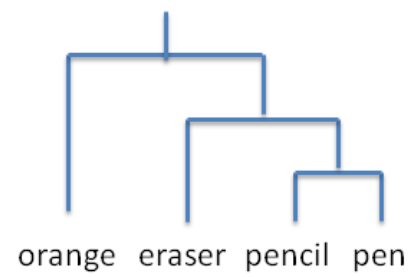

$B$

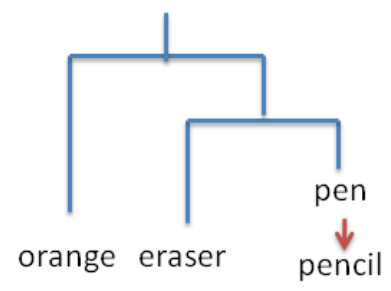

C

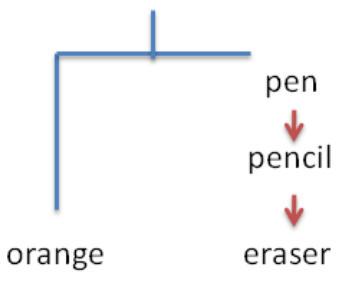

$D$

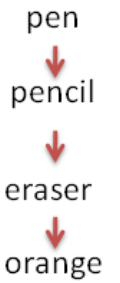

$E$

Figure 3: Simple example of Hierarchy generation, according to Fionn Murtagh's approach.

- axes are selected to have along them, in decreasing order from the first to the $k$ th, the maximum possible variance of the projected elements

The creation of the space is done with the application of different operations over the column profiles matrix, the most important between them is a eigenvalue decomposition applied to translate the cloud of modalities into a different referential system where the axes are organized along the directions of maximum distribution of the points.

Knowing that the axes are ordered according to the variance of the elements, if we want to reduce the complexity of the representation, we can just discard as many dimensions as we want, starting from the last one. In this way, we know that we keep always the dimensions with the highest distribution, so, the dimensions carrying the higher amount of distributional information. Plot in Figure 2 was obtained just keeping the 2 most important axes in the $k$-dimensional space created.

\subsection{Creation of the Hierarchy}

At this step the user of our Extraction tool can choose among different algorithms to be applied for the creation of the hierarchy. The options are four:

- Murtagh's Algorithm.

- Hearst Patterns on Web.

- Maedche and Staab's Bootstrapping.

- An original combination of the last two.

\subsubsection{Murtagh's Algorithm}

This approach applies the technique presented by Fionn Murtagh in (Murtagh, 2007) for the generation of a hierarchy of concepts from a hierarchical clustering representation. Having used the Correspondence Analysis technique, adopted in many works by Fionn Murtagh, it was natural for us to experiment with his solution for the generation of hierarchies of concepts, although better results can be obtained with other techniques.

A Hierarchical Clustering tree is created defining the terms proximities in the tree according to their proximity in the Euclidean space. The algorithm starts from this representation to build the concept hierarchy (see Figure $3, A$ ), ordering clusters from right to left according to their proximity to the origin in the $k$-dimensional representation. This is done because terms appearing closer to the origin are expected to be more general terms, being their occurrences distributed over the maximum number of documents. In our case, the representation is exactly specular to what we had (Figure 3, $B$ ).

Starting from the first created cluster, the right sibling is always considered as a hyponym of the left one. Thus, in our example, pen would be considered as a hypernym of pencil (Figure 3,C). Again, the right siblings, pen and pencil, are hypernyms of the left one, eraser (Figure 3,D). Finally, pen, pencil and eraser are hypernyms of orange (Figure $3, E$ ). This final representation is returned as the searched hierar- 


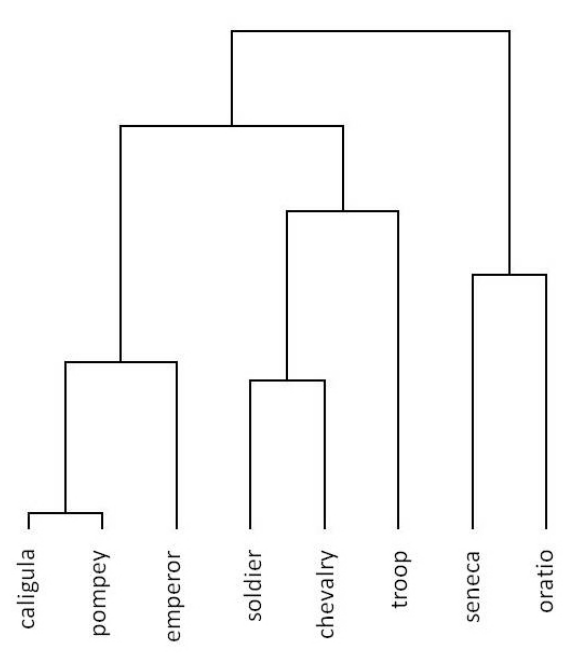

Figure 4: Example of Hierarchical Clustering of terms extracted from the 2-dimentional projection of the corpus about the Roman Empire of Figure 2.

chy. An example of a Hierarchical Clustering tree extracted from Correspondence Analysis depicted in Figure 2 is shown in Figure 4.

\subsubsection{Hearst Patterns on Web}

The Hearst Patterns on Web approach can be applied both for the creation of a hierarchy from scratch or to expand an existing one. We created this solution taking inspiration from the application PANKOW and the general principles of Learning by Googling.

The algorithm starts with a hierarchy to be expanded (bootstrapping), or with a empty hierarchy if we want to create a new one. In the latter case, the first term $t_{1}$ to be added is simply put under the root element. Then, for every new term $t_{n}$ to be added, its lokelihood to be an hyponym of any term $t_{i}$ already in the hierarchy is checked as follows:

1. five different pre-defined strings based on Hearst patterns are built:

- $h p_{1}$ : pluralize $\left(t_{i}\right)$ such as $t_{n}$

- $h p_{2}$ : pluralize $\left(t_{i}\right)$ including $t_{n}$

- $h p_{3}$ : pluralize $\left(t_{i}\right)$ especially $t_{n}$

- hp 4 : pluralize $\left(t_{i}\right)$ like $t_{n}$

- $h p_{5}: t_{n}$ is a/an $t_{i}$

2. six Google queries are executed (the five Hearst patterns plus the single term $t_{n}$ ), obtaining the number of Google hits for each query

3 . the score of every string is defined as the ratio between the number of its Google hits and the number of Google hits of the hyponym searched alone

$$
\text { score }_{h p_{i}}=\frac{\text { googleHits }\left(h p_{i}\right)}{\text { googleHits }\left(t_{n}\right)}
$$

4. the total score is obtained as a sum of the different five scores

Once the hypernymy scores have been calculated for every $t_{i}$ in the hierarchy, $t_{n}$ is placed as hyponym of the $t_{i}$ with the highest score between all the scores, provided that it exceeds a pre-defined threshold level ${ }^{4}$.

If no $t_{n}$ hypernyms exceed the threshold level, the user can possibly suggest a hypernym manually. She can also select to discard the term or to add it in the hierarchy as a hyponym of the root element. The algorithm can also be executed in its automatic mode, in this case it directly puts the $t_{n}$ term as a hyponym of the root element.

After $t_{n}$ is placed, all its siblings are checked in the same manner for an hyponymy relation with $t_{n}$; if the relation surpasses the threshold, the sibling element is put as a hyponym of $t_{n}$.

\subsubsection{Maedche and Staab's Bootstrapping}

Maedche and Staab's Bootstrapping Process is an instantiation of the model defined by A. Maedche and S. Staab in (Maedche A., 2003). In this model, a concept hierarchy is expanded adding a new $t_{n}$ term according to the hypernyms of its $m$ nearest neighbors in the $k$-dimensional space, where $m$ is a parameter of the algorithm. The score for every $f$ candidate hypernym is calculated as follows.

The Least Common Superconcept between two concepts $a$ and $b$ in a hierarchy is defined as:

$$
l c s(a, b)=\underset{c}{\operatorname{argmin}} \delta(a, c)+\delta(b, c)+\delta(\text { root }, c),
$$

where $\delta(a, b)$ is the distance between $a$ and $b$ in terms of the number of edges which need to be traversed. Then the taxonomic similarity $\sigma$ between two concepts in a hierarchy can be defined:

$$
\sigma(a, b)=\frac{\delta(\text { root }, c)+1}{\delta(\text { root }, c)+\delta(a, c)+\delta(b, c)+1},
$$

where $c=l c s(a, b)$. The $W(f)$ score for a certain candidate hypernym $f$ is finally computed as:

$$
W(f)=\sum_{h \in H(f)} \operatorname{sim}\left(t_{n}, h\right) \cdot \sigma(n, h),
$$

where $t_{n}$ is the term to be classified and $H(f)$ is the set of hyponyms of candidate hypernym $f$ that are also nearest neighbors of $t_{n}$ in the $k$-dimensional space.

\footnotetext{
${ }^{4}$ The threshold level was empirically identified after few experiments.
} 
The sim function is the similarity between two concepts as obtained from the $k$-dimensional space.

If no $t_{n}$ hypernyms are found, the user can possibly suggest a hypernym manually. She can also select to discard the term or to add it in the hierarchy as a hyponym of the root element. The algorithm can also be executed in its automatic mode: in this case, it directly puts the $t_{n}$ term as a hyponym of the root element.

\subsubsection{Combination of Hearst Patterns on Web and Bootstrapping Algorithms}

This approach works by combining the two previous algorithms we described for expanding a hierarchy. The pre-existing hierarchy could be huge, and a new $t_{n}$ term to be added with the Hearst patterns algorithm would generate a huge amount of connections to the Google servers. Depending on the Internet connection speed, the execution of a very large number of HTTP requests could be very time consuming.

In this case, we look for the $n$ nearest neighbors in the $k$-dimensional space of $t_{n}$ in the pre-existing hierarchy, and collect all their ancestors. These ancestors are considered the candidate hypernyms of $t_{n}$ and they are checked according to the Hearst Patterns on Web algorithm.

If no hypernyms are found, the user can possibly suggest a hypernym manually. She can also select to discard the term or to add it in the hierarchy as a hyponym of the root element. The algorithm can also be executed in its automatic mode, in this case it directly puts the $t_{n}$ term as a hyponym of the root element.

\section{TESTS AND RESULTS}

We evaluated the precision of the ontologies generated by some of the automatic and assisted algorithms, defined as the ratio between the right relationships (as evaluated by a human judge) and the totality of the relationships found:

$$
\text { precision }=\frac{\text { right relationships }}{\text { total relationships in ontology }}
$$

Three different corpora of documents were selected as test corpora:

- a set of 847 Wikipedia articles about Artificial Intelligence and related arguments;

- a set of 1464 Wikipedia articles about Roman Empire and related historical articles;

- a set of 1364 Wikipedia articles about Biology.

As a referential Training corpus we always use the same collection of 1414 Wikipedia articles about
Wikipedia itself. This choice was made according to what we stated about the Information Gain measure for term relevance: we expected from the comparison between any of the three test corpora and this Wikipedia training corpus that less importance would have been given to terms typical of every Wikipedia article. This, in fact, proved to be correct and terms as "Wikipedia", "Wikimedia", or "article", that have a high frequency in all the four corpora of documents, received a lower Information Gain value with respect to other characterizing terms of each test corpus.

We then applied the Correspondence Analysis algorithm in order to generate a 2-dimensional representation of distributional similarity of the relevant terms, starting from this representation to execute the different ontology learning algorithms described in this paper. In Table 1 the average precision measures of the ontologies obtained from the application of the algorithms are summarized.

While Murtagh's algorithm does not seem to perform well, the research for Hearst patterns via Web seems to be a good option for the generation of concept hierarchies. Its semi-automatic version provides nearly ready-to-go ontologies, producing hierarchies such as the one depicted in Figure 5.

Maedche and Staab's Bootstrapping algorithm and its combination with Hearst Patterns on Web algorithm were designed to expand large concept hierarchies, and, as we did not have one available, no valid attempts have been done to test them. Early tests with small ontologies, anyway, showed that the combination of Maedche and Staab's algorithm with Hearst Patterns on Web improves the precision of Maedche and Staab's algorithm alone of about $10 \%$, but these should be considered as preliminary results so are not reported in this paper.

\section{CONCLUSIONS}

In this paper we presented Extraction, a tool for the generation of seed ontologies from text. Addressing the problem of ontology extraction from text, we developed a modular system whose main advantage, in our opinion, is the original co-presence of the following three features:

- a pre-process of identification of relevant terms, which relies on the calculation of information gain to reduce the complexity of the following elaboration steps;

- the strong framework of correspondence analysis for the computation of distributional similarity between terms; 
Table 1: Precision measures obtained from the evaluation of the different ontology learning algorithms.

\begin{tabular}{|l|c|c|c|c|}
\hline Algorithm & AI & Rome & Biology & Average \\
\hline Murtagh & $6.6 \%$ & $5.22 \%$ & $1.87 \%$ & $\mathbf{4 . 5 6 \%}$ \\
Hearst Patterns on Web & $60.00 \%$ & $75.00 \%$ & $37.50 \%$ & $\mathbf{5 7 . 5 0 \%}$ \\
Hearst Patterns on Web (assisted) & $90.00 \%$ & $94.52 \%$ & $85.07 \%$ & $\mathbf{8 9 . 8 6 \%}$ \\
\hline
\end{tabular}

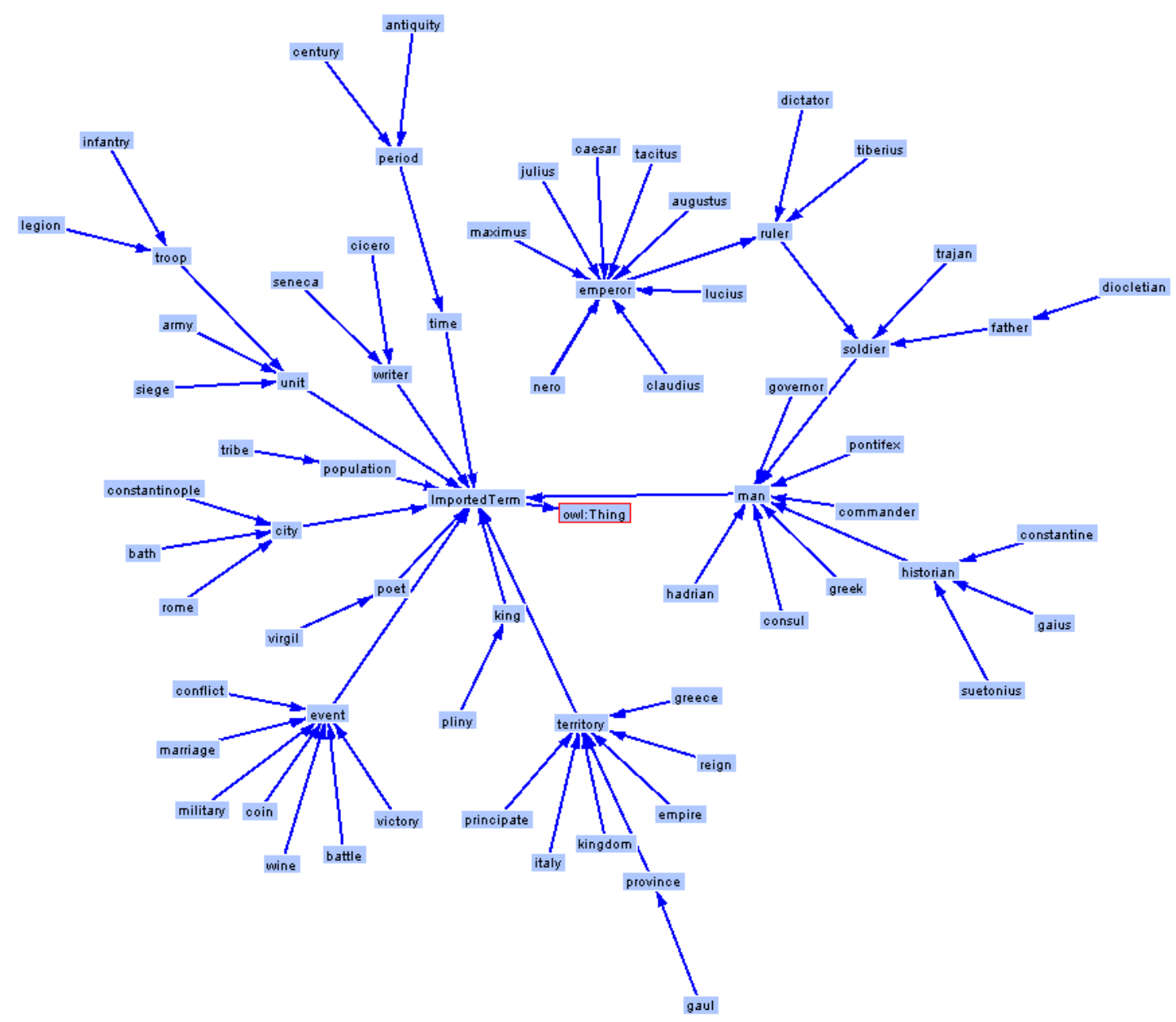

Figure 5: A hierarchy created by the Hearst Patterns on Web algorithm in its semi-automatic procedure, from 100 terms of a corpus about the Roman Empire.

- the possibility to use different algorithms for the generation of ontologies.

The results of our evaluation are promising. The tool surely represents an evolution and an improvement in performance with respect to Murtagh's approach, that uses an algorithm for the generation of hierarchies which strongly depends on an inherent characteristic of texts called ultrametricity (Murtagh, 2007), condition which does not appear to be met in the documents that we have analyzed. The assisted Hearst patterns method, despite of having the disadvantage of requiring user interaction, provides highquality hierarchies which are almost ready to use. Fi- nally, the tool allowed us to combine different algorithms and provide qualitatively better results, such as in the case of Maedche and Staab's plus Hearst patterns.

As a negative note, while making the tool more powerful by adding functionalities based on NLP we also made it more dependent on a specific language. This is a limitation if we compare our approach to Murtagh's, as pure correspondence analysis is language independent. However, this limit is somehow counterbalanced by the advantages that NLP provides, such as the possibility of filtering extracted concepts by keeping only names without the need to 
rely on a limited vocabulary like Wordnet. Similarly, the extraction of concepts with information gain depends on the set of documents that is chosen as a training corpus: while this obviously looks like a limitation, it also has useful drawbacks like in our evaluation, when we were able to use a set of general Wikipedia documents to automatically filter all the Wikipedia-related text from the articles we wanted to study.

For what concerns possible future improvements to our project, we would like to continue our work in two main directions: on the one hand, improving our evaluations by testing our tool with large ontologies (especially while using the bootstrapping algorithm) and comparing its results with the ones obtained by using other algorithms; on the other hand, improving the application itself by adding new functionalities and a better user interface to easily configure them.

\section{REFERENCES}

Alfonseca E., M. S. (2002). Extending a lexical ontology by a combination of distributional semantics signatures. In Proceedings of the 13th International Conference on Knowledge Engineering and Knowledge Management.

Benzcri, J.-P. (1976). L'Analyse des Donnes. Dounod.

Caraballo and Charniak (1998). New figures of merit for best-first probabilistic chart parsing. In Computational Linguistics.

Caraballo, S. (1999). Automatic construction of a hypernym-labeled noun hierarchy from text. In Proceedings of the 37th Annual Meeting of the Association for Computational Linguistics.

Cimiano, P. (2006). Ontology Learning and Population from Text. Springer.

Cimiano P., Handschuh S., S. S. (2004). Towards the selfannotating web. In Proceedings of the 13th World Wide Web Conference.

Faure D., N. C. (1998). A corpus-based conceptual clustering method for verb frames and ontology. In Proceedings of the LREC Workshop on Adapting lexical and corpus resources to sublanguages and applications.

Harris, Z. (1968). Mathematical Structures of Language. Wiley.

Hearst, M. (1992). Automatic acquisition of hyponyms from a large text corpora. In Proceedings of the 14th International Conference of Computational Linguistics.

Hearst M., S. H. (1993). Customizing a lexicon to better suit a computational task. In Proceedings of the ACL SIGLEX Workshop on Acquisition of Lexical Knowledge from Text.

Maedche A., Pekar V., S. S. (2003). On discovering taxonomic relations from the web. Technical report, Institute AIFB - University of Karlsruhe, Germany.
Murtagh, F. (2005). Correspondence Analysis and Data Coding with Java and $R$. Chapman \& Hall.

Murtagh, F. (2007). Ontology from hierarchical structure in text. Technical report, University of London Egham.

Schutze, H. (1993). Word space. In Advances in Neural Information Processing Systems 5. 